\title{
Clues for stellar formation in NGC 1275 cooling flow
}

\author{
Pierre Ferruit and Emmanuel Pécontal \\ Observatoire de Lyon, 69561 St-Genis-Laval CEDEX, France
}

\begin{abstract}
D spectrography of the inner regions of NGC 1275 has been performed using the integral field spectrograph TIGER. It revealed an association between star clusters and some line emitting gas components, likely to be filaments material. This may indicates that the clusters originate from star formation in the cooling flow
\end{abstract}

\section{The Zoo...}

NGC 1275, the central cD galaxy of the Perseus cluster is some kind of zoo for physical phenomena (see Fig. 1). Indeed, one can find there : a) an active galactic nucleus exhibiting a strong nuclear continuum source and Broad and Narrow Line Regions (BLR and NLR hereafter). This activity is associated with a strong nuclear radio source (3C84) and a jet (Pedlar et al. 1990). b) a prototypical cooling flow (see reviews of Fabian 1987; Baum 1992) traced by the diffuse X-ray emission and a complex system of $\mathrm{H} \alpha$ emitting filaments (Lynds 1970) likely to originate from the cooling and condensation of the intracluster medium. c) a merger with a $+3000 \mathrm{~km} \mathrm{~s}^{-1}$ infalling foreground galaxy (Rubin et al. 1977) called the High Velocity (HV) system. d) The last (but not least) comer in this already crowded place, is a population of blue stars clusters discovered by Holtzman et al. (1992) using the HST.

We will mainly focus on the latter whose origin is very debated. Indeed, Holtzman et al. (1992) found from their images (using colors diagrams) that the cluster ages distribution was quite uniform and well explained by a merge between NGC 1275 and a second galaxy some $10^{8}$ yr ago. This was welcomed for the scenario of formation of elliptical galaxies through merger events. But, later ground based observations favour the idea of the clusters being formed in the cooling flow (Richer et al. 1993), and point out a much more diversified population (Norgraard-Nielsen et al. 1993). In fact, the crowded environment, the steep variations of the background and uncertainties in the extinction corrections in this galaxy can easily account for these discrepancies.

\section{The TIGER and the Zoo...}

Bidimensional spectrography of the central regions of NGC 1275 has been performed with TIGER (see paper of R. Bacon in this colloquium for a description of the instrument) at the $3.6 \mathrm{~m}$ CFH Telescope. We acquired two half an hour spectrographic exposures in the [NII], H $\alpha,[\mathrm{SII}]$ domain (filter 6750/500 $\AA$ ) with 


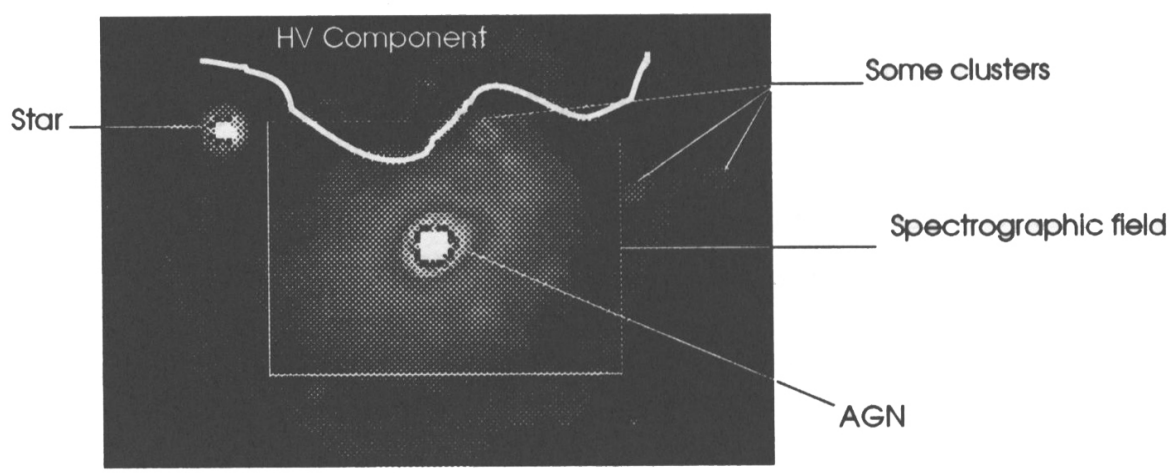

Figure 1. TIGER direct image (filter $6750 / 500 \AA$ ) of NGC 1275 with the location of several tenant of the zoo

a 3.6 $\AA$ spectral resolution. The $11^{\prime \prime} \times 7^{\prime \prime}$ spatial field is centered on the nucleus and has a sampling of $0.39^{\prime \prime}$ yielding to a resolution of $0.8^{\prime \prime}$. A complete description of the data set and of its reduction is given in Ferruit and Pécontal (1994).

\section{Whos's Who in the Zoo...}

In this very crowded environment, the superposition of various phenomena makes the analysis of the data very difficult. But, one feels much more comfortable when disposing of a 3D data cube which allows to gather spectral and spatial information. To identify and disentangle the different contributions, we first separate the continuum from the emission lines using polynomial fitting. The reconstructed continuum map (see Fig. 2) displays : the nuclear component, a bar-like structure close to the nucleus, a diffuse component and the clusters contribution. From the continuum subtracted spectra, we identify at least five contributions : the BLR, the NLR, the diffuse narrow emission component (systemic component hereafter), the $\mathrm{HV} \mathrm{H \alpha}$ line and some additional components. The BLR and the NLR were assumed to be spatially unresolved and their spectra subtracted using the weights of the point spread function. We then performed a fitting of the lines of the systemic component using gaus. sian profiles, adding line systems when needed (for the HV system, additional components...).

\section{The unexpected guests}

As shown in Fig. 2, the spatial repartition of the additional components is very closely related to the location of the brightest clusters found in our field. Moreover, they might not be part of the systemic component, as they have velocities $\left(-380 \mathrm{~km} \mathrm{~s}^{-1}\right.$ and $\left.+270 \mathrm{~km} \mathrm{~s}^{-1}\right)$ outside of the range found for it $(-160$ to $\left.+160 \mathrm{~km} \mathrm{~s}^{-1}\right)$. This suggest that this morphological correlation is not the result of a fortuitous superposition, but reflects a real link between this line emitting 



Figure 2. Continuum reconstructed map with the isophotes of the additional components overimposed.

gas and the clusters.

The line widths (320 and $520 \mathrm{~km} \mathrm{~s}^{-1}$ ) and the line ratios (liner type spectrum) imply that the gas is neither gravitationally bound to the clusters, nor photoionized by their stars, and so is unlikely to be internal cluster gas. But, its spectral and kinematical characteristics do agree with that expected from filaments material. This association is naturally explained if the clusters have formed in the filaments, i.e. in the cooling-flow.

This favours the scenario where the clusters originate from star formation in the cooling-flow, likely to be continuous, but raises many questions : a) Where are the young clusters? They should be easy to identify owing to their HII region type spectra (except if the IMF is biased toward the low mass stars). b) Why some clusters in our field are not associated with line emitting gas ? Are they older than the filaments life-time, have they formed in gas-poor filaments which are below our detection limit? 


\section{From clues to evidences ?}

Our present observations point out clues of an association between the clusters and gas of the filaments. We will observe NGC 1275 again with TIGER in november 1994 with : a) a larger spatial field to complete our sample of observed clusters b) both $\mathrm{H} \beta$, [OIII] and [NII], $\mathrm{H} \alpha,[\mathrm{SII}]$ domains, yielding to a more complete kinematical and spectral information, and allowing to compute color gradients $\mathrm{c}$ ) longer integration times, to increase $\mathrm{S} / \mathrm{N}$ ratio and lower the detection limit. This new set of data should make clues become evidences... It is worth noticing that most of our results (not only those dealing with the clusters, see Ferruit and Pécontal 1994) are unreachable without 3D spectrography!

\section{Discussion}

H. Hippelein: Concerning the two star-forming regions in the filaments, how do you know it is inflowing and not outflowing gas? Can you prove the cooling-flow from your data?

$P$. Ferruit: No, we don't know if the gas is in front or behind the galaxy. We have assumed that the gas was inflowing in a cooling flow.

R. Gelderman: : Was the map of $\mathrm{H \alpha}$ emission, shown with the [NII] map, only narrow line emission (BLR component subtracted)?

$P$. Ferruit: It is a map of the sytemic component, BLR and NLR subtracted.

$R$. Gelderman: Do you have an estimate for the limiting surface brightness limits of your emission line observations?

P. Ferruit: Yes, our detection limit $(3 \sigma)$ was $1.410^{-19} \mathrm{Wm}^{-2} \AA^{-1} \operatorname{arcsec}^{-2}$.

\section{References}

Baum, S.A. 1992, in the proceedings of the NATO/ASI school on "Clusters and Superclusters"

Fabian, A.C. 1987, in "Starbursts and galaxy evolution", Editions Frontières, 355

Ferruit, P., and Pécontal, E. 1994, A\&A, in press

Holtzman, J.A., et al. 1992, AJ, 103 (3), 691

Lynds, C.R. 1970, ApJ, 159, L151

Norgaard-Nielsen, H.U., et al. 1993, A\&A, 279, 61

Pedlar, A., et al. 1990, MNRAS, 246, 477

Richer, H.B., et al. 1993, AJ, 105 (3), 877

Rubin, V.C., et al. 1977, ApJ, 211, 693 